\title{
Elevator Regenerative Energy Feedback Technology
}

\author{
Peng Gao ${ }^{\text {a }}$, Weifei Niu, Zhuojun Quanji, Yang Yang and Yinghui Lv \\ Tianjin special equipment inspection institute, Tianjin 300192, China \\ apenggao@tju.edu.cn
}

Keywords: Elevator, Regenerative energy feedback, Regenerative energy storage.

\begin{abstract}
Elevator regenerative energy feedback technology is an important method of reducing energy consumption. Elevator regenerative energy feedback technology includes energy feedback system structures and feedback energy storage methods. This article introduces the feedback system structures and energy storage methods. The dual PWM regenerative energy feedback circuitry and plug-in regenerative energy feedback system are analyzed, and their different characteristics are concluded; the battery and the supercapacitor energy storage methods are discussed and the novel technology on the energy storage is described. This paper aims to introduce different structures and storage methods to help deepen the further understanding on the elevator energy feedback technology.
\end{abstract}

\section{Introduction}

With the continuous development of smart grid technology, as well as the reliability increased of wind, solar and other renewable energy sources and power costs decreased, people try to reduce the use of fossil fuels and carbon dioxide emissions [1, 2]. Meanwhile, people are also concerned about energy efficiency, in order to increase the efficient utility of energy. In the modern high-rise building, the elevator is a kind of high energy consumption transport equipment. Elevators typically account for about 3 to $8 \%$ of the overall electricity consumption of a building [3]. Elevator energy conservation is also an important factor in building energy saving. Some mature elevator energy saving methods have been used in our life: Traditional worm gear are changed to gearless; LED lamps are used in the elevator car to reduce elevator lighting energy consumption; Additional, intelligent elevator control technologies are adopted, such as auto sleep, auto power off, and elevator group control systems [4, 5].

Elevators are important vertical transportation equipments with the total weight of the car and predetermined passenger balancing with the counter weight. If an imbalance occurs because the total weight of the passengers and the car is heavier while descending, the traction motor regenerates electric power. Likewise, if the total weight of the passengers and the car is lighter while ascending, the traction motor will also regenerates electric power. For traditional elevator, regenerative power generated by the traction motor either consumed in the motor windings or in the brake resistors. The regenerative power consumed in the brake resistors results in electrical energy waste and temperature increment in elevator machine room. In the condition of temperature increment, additional installation of air conditioning is needed, which further increases the energy consumption. Using the inverter working principle of AC-DC-AC, the renewable energy is converted into the form of direct current. Through the current inverter technology, DC is inverter into AC back into the grid. The renewable energy can be used by other electric equipment, so that the power consumption falls and the energy saving of the elevator are realized.

In this paper, elevator energy feedback technology research and development is presented. The review includes feedback system scheme and energy storage mode, which helps deepen the further understanding on the elevator energy feedback technology. 


\section{Scheme of the energy feedback system}

\subsection{Double-side PWM energy feedback scheme}

The double-side PWM converter consisting of two full-bridge converters has the capacity of bidirectional energy flow [6], through on-off control of the switching devices in the inverter in accordance with the algorithm has been set $[7,8]$. Double-side PWM converter system works in four-quadrant operation with least harmonics and high power factor, which is one of the high-quality energy feedback technologies [9, 10]. At present, only a few companies, such as Otis and Mitsubishi, have this kind of products in the world.

Fig. 1 shows the basic scheme of double-side PWM converters which including choke reactor, input converter (grid side converter), output converter (motor side converter) and control circuit. The three-phase diode rectifier bridge is replaced with 6 full-controlled power switching device IGBTs.

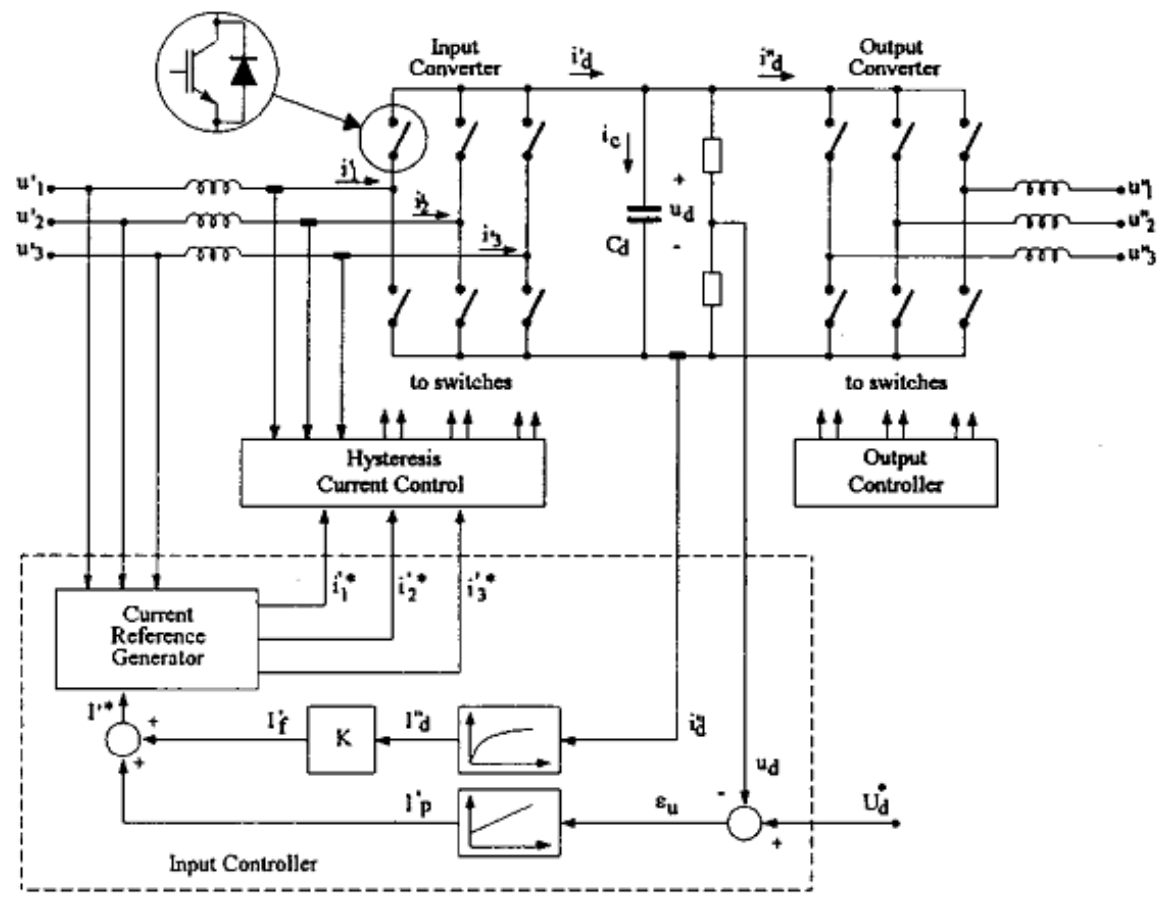

Fig. 1. Basic scheme of double-side PWM converter [6]

When the elevator motor is in motor-operated running stage, the grid will supply energy to the motor. The grid side converter and the motor side converter operate as rectifier and inverter respectively. The synchronization with a grid is controlled by the grid side converter using PWM. Motor-side converter output voltage is proportional to operating frequency in the PWM control mode that makes the AC motor show constant torque characteristics. Input current to the motor is a sine wave, reducing the loss of high harmonic currents [6].

When the elevator traction motor is in generator-operation stage, the grid side converter will work in the inverter mode. The inverter transform the energy generated by traction motor, which gathered in the DC capacitor, into alternating current (AC) back to the grid, in order to maintain a constant voltage in DC bus. The system obtains near-unity power factor.

\subsection{Plug-in energy feedback scheme}

Plug-in energy feedback system is based on AC-DC-AC traction control system, with energy feedback device in parallel with the DC bus, and reverses the regenerated power from the DC bus to the power grid, which is shown in Fig.2. The elevator consumes or produces electric energy depending on the moving direction and the load conditions. When the elevator goes up with light load, the car is lighter than the counter weight, so the elevator is in generator-operation stage. The energy was transformed from mechanical energy to electric energy. When the elevator is in generator-operation stage, the voltage of the DC bus increases, and the voltage on the electrolytic capacitor increases simultaneously. If the DC bus voltage reaches the set-point value of the upper limit, the three-phase full-bridge inverter begins to work and reverses the regenerated energy to the 
power grid, so the voltage of the DC bus decreases. If the DC bus voltage decreases to the set-point value of the lower limit, the three-phase full-bridge inverter stops working. That means that the feedback device works only when the elevator is in generator-operation stage and stops working when the elevator is in motor-operation stage or stop stage.

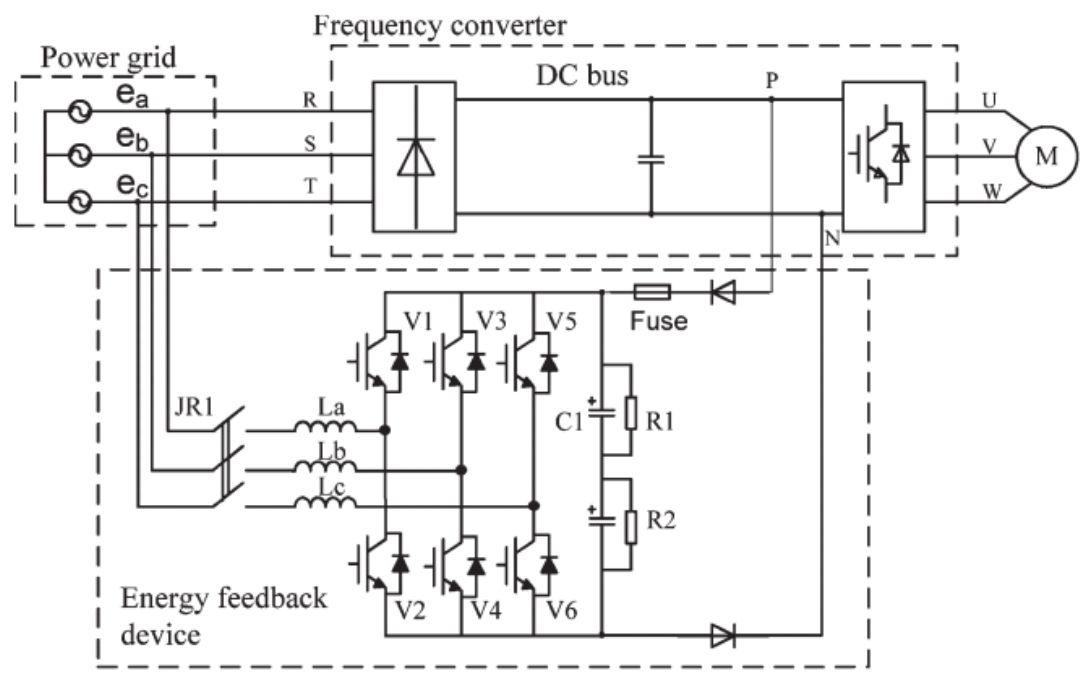

Fig. 2. Main circuit of the plug-in feedback system connected with a frequency converter

\section{Energy storage}

\subsection{Energy storage with battery}

Since the feedback energy change frequently at different time points, the feedback energy could destabilize the grid. For some cases that feedback energy cannot be transform to the grid directly, the feedback energy can be stored. When there is insufficient power supply, the stored feedback energy can be transformed to the grid, which helps balance power grid. The researchers developed a type of elevator with battery energy storage (BES) devices $[11,12]$. With the battery energy storage devices, the feedback energy can be storged. The batteries discharge energy in motor-operation state for the elevator. Shinji Tominaga et al used nickel metal hydride (Ni MH) batteries for renewable feedback energy storage of elevator at night. Fig. 3 shows the system configuration of the energy-saving elevator using regenerated power storage system. The designed prototype system were verified and a good result was obtained [11].

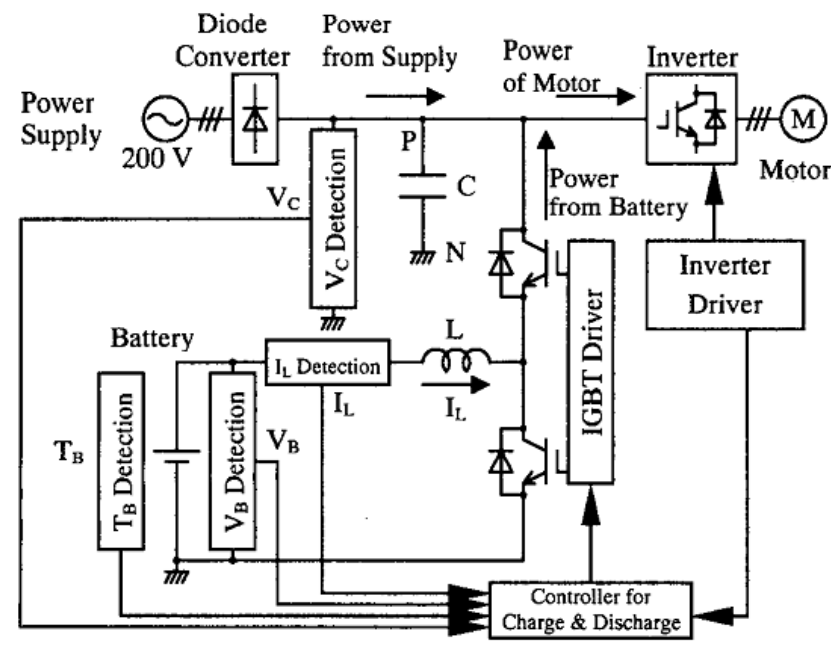

Fig.3 System configure [11]

Shili Lin et al developed a similar energy storage system based on batteries. The battery energy storage system (BESS) insisting of Li4Ti5O12 (LTO)-based batteries is put forward in order to suppress the voltage fluctuation of the DC grid of elevator caused by regeneration energy of the 
traction machine. The topology structure of BESS used in the power supply system of the elevator is shown as Fig. 4, in which the battery pack that is made up of many SCiB cells by the way of series-parallel connection is linking to the DC grid via a bidirectional DC/DC converter. Through controlling the switch state of the converter, the BESS could absorb or release the regeneration energy and achieve the stabilization of the DC grid voltage [13].

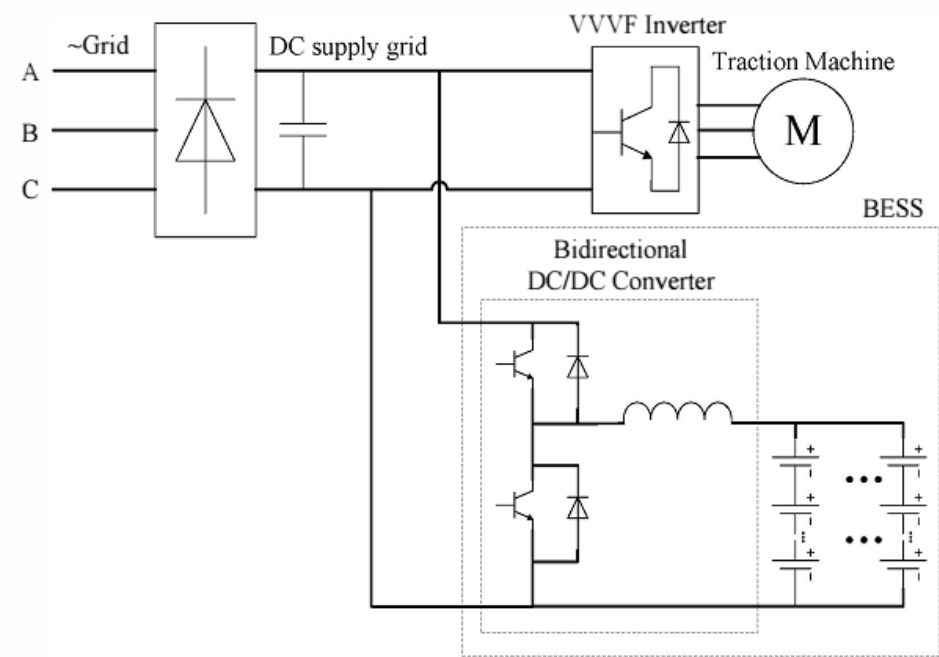

Fig. 4 Topology structure of BESS in power supply grid of elevator [13]

The feedback scheme using the battery energy storge system could absorb or release the regeneration energy through controlling the switch state of the converter. But there are some shortcomings in the battery energy storge system. The structure needs the batteries to have a greater voltage range than the DC bus, which can cause excessive much more battery cells, therefore resulting in higher costs, and the battery charge-discharge effeciency is very low.

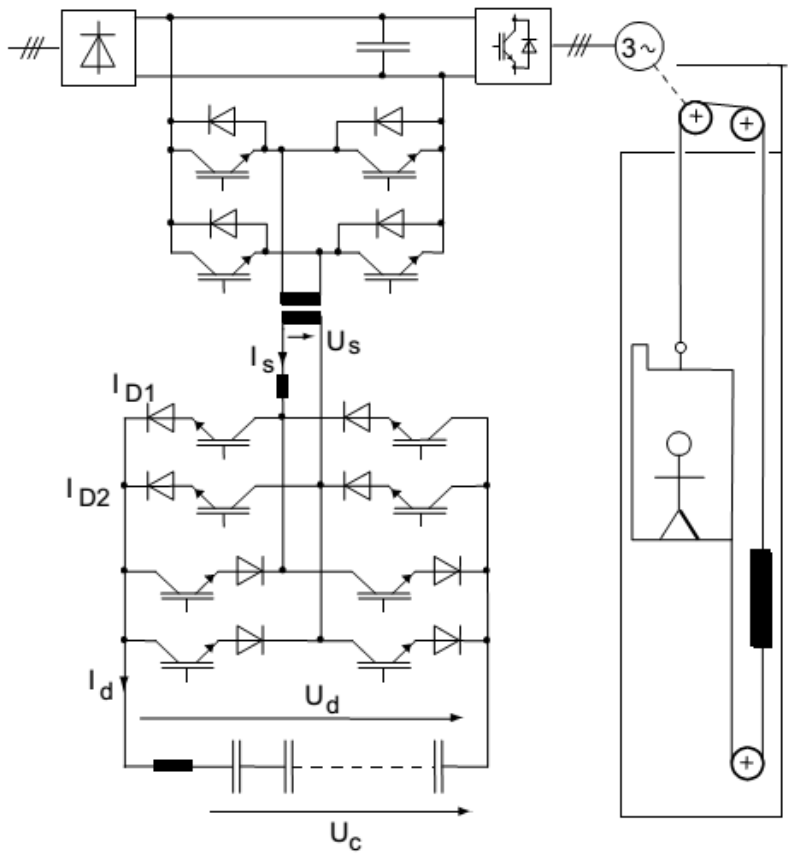

Fig. 5 scheme of the elevator-drive with supercapacitor storage [14]

\subsection{Energy storage with Supercapacitor Bank}

To improve elevator energy-saving technologies in the power shortage, researchers proposed a supercapacitor technology solution to replace the battery energy storage devices. The scheme of supercapacitor-based technology is similar to the battery-based one, in which the supercapacitor bank is linking to the DC grid via a bidirectional DC/DC converter. The bidirectional DC/DC converter is the core component of the scheme and the researchers have studied a variety of converters in recent 
years including soft commutated interface [14], Buck-boost [15], and multichannel Buck-boost bidirectional DC/DC converter.

Alfred Rufer et al proposed a scheme of the elevator-drive with supercapacitor storage. A high efficient converter topology has been proposed, which allows the bidirectional energy-flow with soft-commutation. That solution offers also a good flexibility for the optimal sizing of the supercapacitor voltage level, and reduces the number of components in the load equalizing circuitry of the series-connected supercapacitors. Power Electronic circuits with current controlled mode are unavoidable for reaching an acceptable efficiency in the charging and discharging cycle of supercapacitive tanks [14].

Zheng Li et al proposed a new energy storge scheme, in which the bidirectional DC-DC converter was used to control the power flow. A novel energy saving control strategy with fuzzy logic controller was proposed in the ride-through system. In their simulation case, $58.6 \%$ of the feedback energy has been recycled [15]. The bi-directional converter with fuzzy controller is shown in Fig. 6.

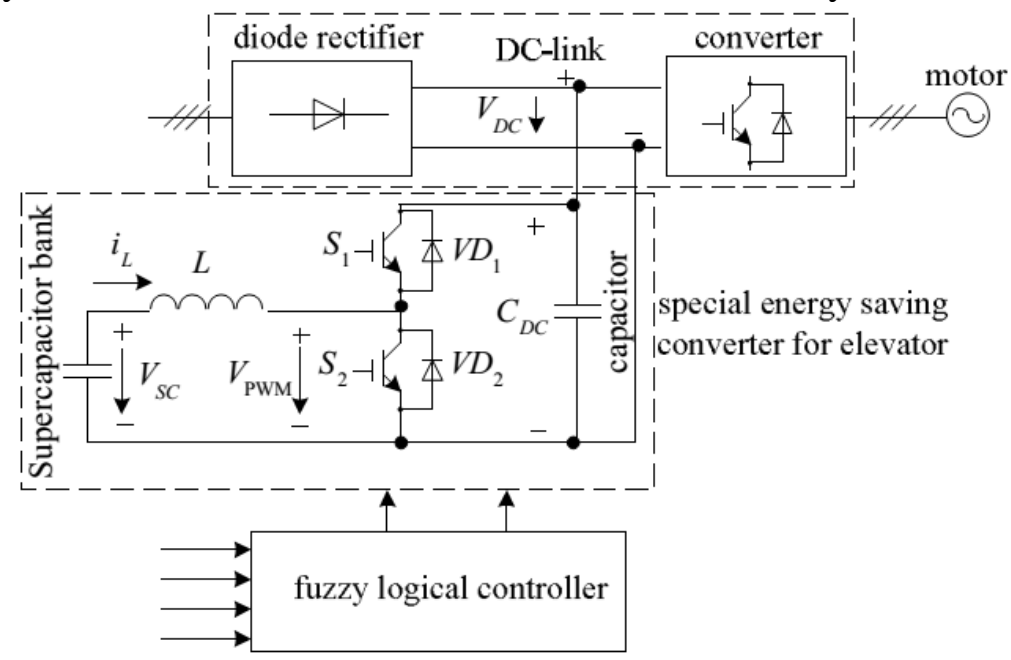

Fig. 6 Bi-directional converter with fuzzy controller [15]

Attaianese et al, from University of Cassino, have developed a scheme based on supercapacitor with bidirectional DC-DC converter. In their design, a power factor correction (PFC) was added, which allowed to greatly improve the elevator performances in terms of energy efficiency and power demand. The proposed conversion system is shown in Fig. 1. The expriment results are given show that the PFC circuit has significantly improved power factor on the grid-side [16].

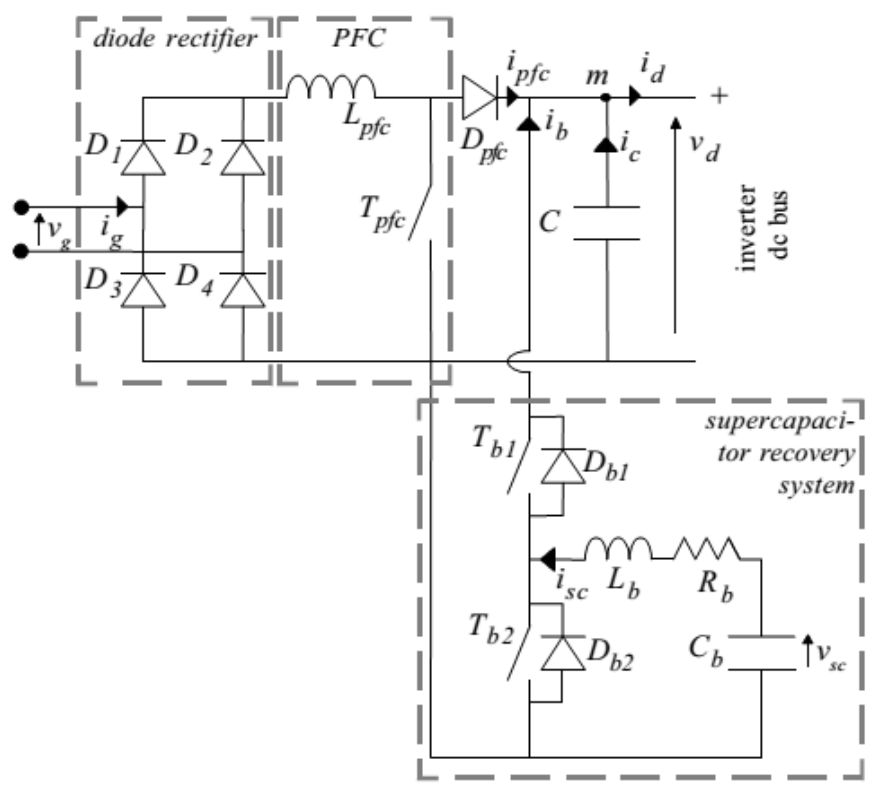

Fig. 7 Conversion scheme with power factor correction [16] 
Nikolaos Jabbour et al employed energy storage system based on supercapacitor bank to improve the conventional elevator. The structure of the proposed elevator system is shown in Fig. 8. The grid supplies energy to the motor in combination with supercapacitors; however, if the supercapacitors are fully charged they can provide energy to the motor autonomously. The supercapacitors are initially charged from the grid and, when the elevator works in power generation state, the supercapacitors are charged from the braking energy of the electric machine [17].

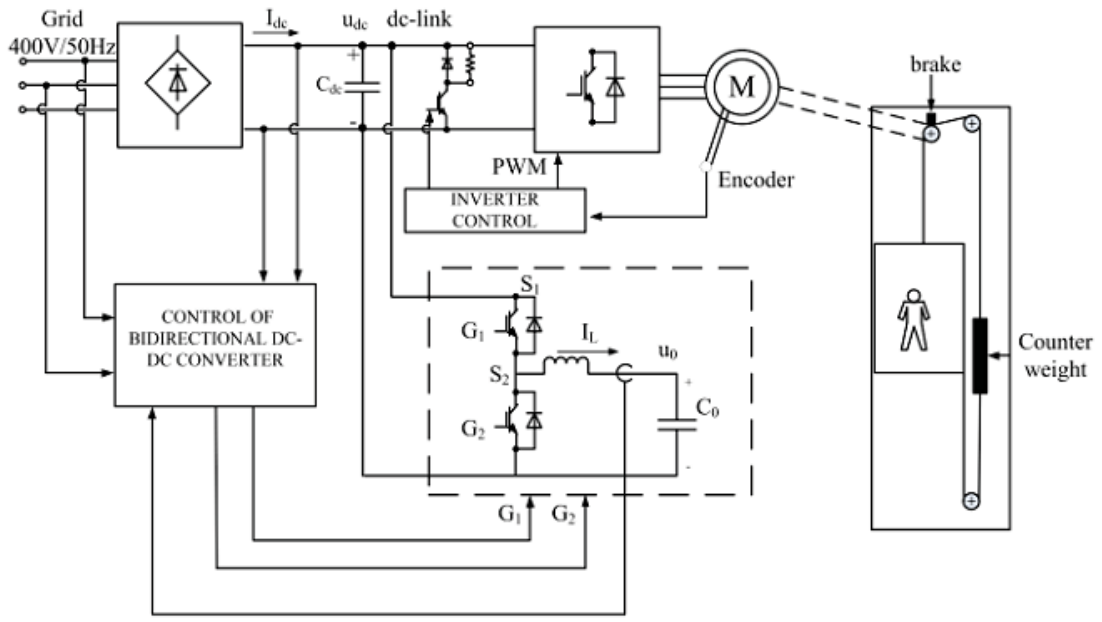

Fig. 8 Structure of the proposed elevator system. The braking energy is stored to a supercapacitor bank that is controlled through a bidirectional DC-DC converter [17]

Sergio Luri et al presented a supercapacitor based energy storage system (SESS) which has been developed based on a multichannel buck-boost converter. Comparing with a conventional elevator system, the designed device is able to achieve an improved efficiency, reducing grid-absorbed power and guaranteeing automatic rescue. A series of simulations and experimental results are presented in their reseach (both in laboratory and in a real elevator), obtained with the developed prototype, which fully validate the design of the energy storage bank, the power converter and the control strategies for the different operation modes. As a result, it has been shown that the SESS can be a very interesting solution for reducing the energy consumption of elevators, reducing grid power and providing added value services such as passenger rescue [18].

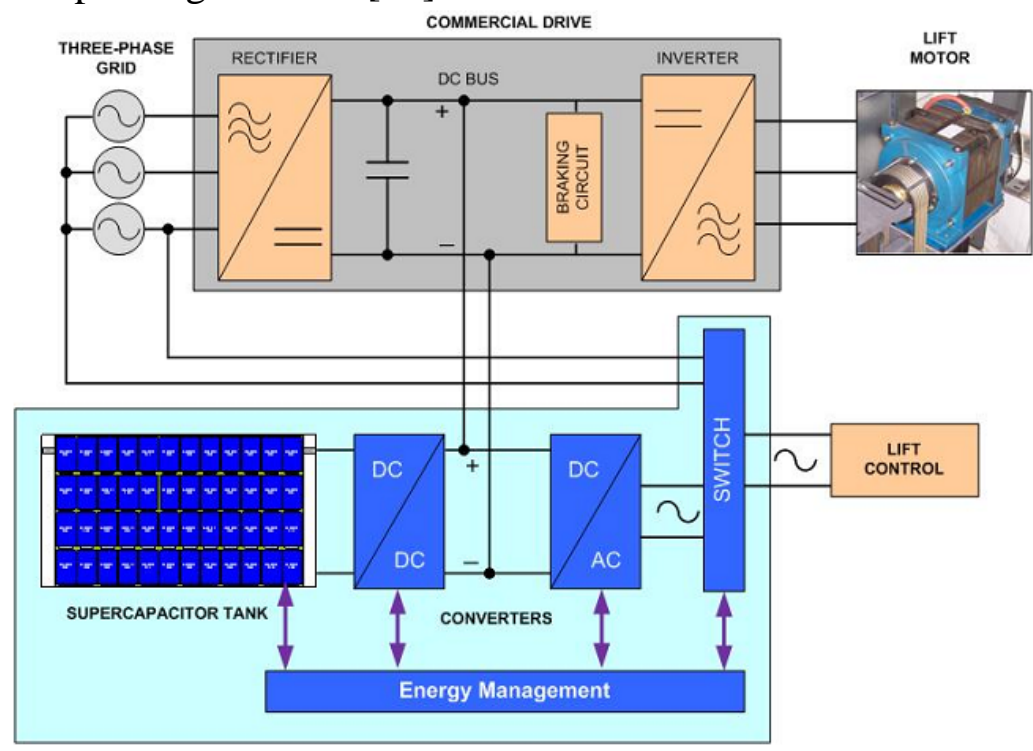

Fig. 9 Elevator system with energy storage in Sergio Luri’s design [18]

\section{Conclusion}

The elevator energy feedback is the most impressive technology in the elevator saving energy reseach field. The elevator equipped with energy feedback inverter feedback the DC bus power into 
the grid through the added inverter device, which avoids feedback energy direct consumption on the the discharge resistance in DC side, achieving the goal of the energy-saving of elevator. Since the feedback energy change frequently at different time points, the feedback energy could destabilize the grid [19]. For some cases that feedback energy cannot be transform to the grid directly, the feedback energy can be stored. The supercapacitor or battery based energy storage system has been developed. Comparing with a conventional elevator system, the elevatior equipped with energy feedback inverter is able to achieve an improved efficiency, reducing grid-absorbed power and guaranteeing automatic rescue, which increases the reliability of the elevators.

\section{Acknowledgments}

This work was supported by The Key Technology R\&D Program of Tianjin, China (Grant No. 14ZCDGGX00793)

\section{References}

[1] Shin-Yeu, L. and C. Jyun-Fu, Distributed optimal power flow for smart grid transmission system with renewable energy sources. Energy, 2013. 56: p. 184-192.

[2] Cecati, C., C. Citro, and P. Siano, Combined operations of renewable energy systems and responsive demand in a smart grid. IEEE Transactions on Sustainable Energy, 2011. 2(4): p. 468476.

[3] De Almeida, A., S. Hirzel, C. Patrao, et al., Energy-efficient elevators and escalators in Europe: an analysis of energy efficiency potentials and policy measures. Energy and Buildings, 2012. 47: p. 151-158.

[4] Afonin, V.I., O.V. Kruglikov, and R.V. Rodionov, Energy consumption of elevator electric drives with different control systems. Russian Electrical Engineering, 2011. 82(3): p. 121-125.

[5] Hasan, M.Z., R. Fink, M.R. Suyambu, et al. Assessment and improvement of intelligent controllers for elevator energy efficiency. In 2012 IEEE International Conference on Electro/Information Technology (EIT 2012), 6-8 May 2012. 2012. Piscataway, NJ, USA: IEEE.

[6] Malesani, L., L. Rossetto, P. Tenti, et al., AC/DC/AC PWM converter with reduced energy storage in the DC link. IEEE Transactions on Industry Applications, 1995. 31(2): p. 287-292.

[7] Gu, B.G. and K. Nam, A DC-link capacitor minimization method through direct capacitor current control. IEEE Transactions on Industry Applications, 2006. 42(2): p. 573-581.

[8] Hur, N., J. Jung, and K. Nam, A fast dynamic DC-link power-balancing scheme for a PWM converter-inverter system. Industrial Electronics IEEE Transactions on, 2001. 48(4): p. 794-803.

[9] Inaba, H., K. Hirasawa, T. Ando, et al., Development of a high-speed elevator controlled by current source inverter system with sinusoidal input and output. IEEE Transactions on Industry Applications, 1992. 28(4): p. 893-899.

[10]Dae-Woong, C., R. Hyung-Min, L. Young-Min, et al., Drive systems for high-speed gearless elevators. IEEE Industry Applications Magazine, 2001. 7(5): p. 52-56.

[11] Tominaga, S., I. Suga, H. Araki, et al. Development of energy-saving elevator using regenerated power storage system. In Proceedings of Power Conversion Conference - Osaka PCC 2002, 2-5 April 2002. 2002. Piscataway, NJ, USA: IEEE.

[12] Desdouits, C., M. Alamir, V. Boutin, et al. Multisource elevator energy optimization and control. In 2015 European Control Conference (ECC), 15-17 July 2015. 2015. Piscataway, NJ, USA: IEEE.

[13] Shili, L., S. Wenji, C. Yongzhen, et al. Study on the model of elevator regeneration energy and its energy storage control method. In 2015 International Conference on Smart Grid and Clean Energy Technologies (ICSGCE), 20-23 Oct. 2015. 2015. Piscataway, NJ, USA: IEEE.

[14]Rufer, A. and P. Barrade, A supercapacitor-based energy-storage system for elevators with soft commutated interface. IEEE Transactions on Industry Applications, 2002. 38(5): p. 1151-9. 
[15]Zheng, L. and R. Yi. A novel energy saving control system for elevator based on supercapacitor bank using fuzzy logic. In 2008 11th International Conference on Electrical Machines and Systems (ICEMS 2008), 17-20 Oct. 2008. 2008. Piscataway, NJ, USA: IEEE.

[16] Attaianese, C., V. Nardi, and G. Tomasso. A high efficiency conversion system for elevators. In IEEE International Conference on Clean Electrical Power, 2007. ICCEP '07, 21-23 May 2007. 2007. Piscataway, NJ, USA: IEEE.

[17] Jabbour, N., C. Mademlis, and I. Kioskeridis. Improved Performance in a Supercapacitor-Based Energy Storage Control System with Bidirectional DC-DC Converter for Elevator Motor Drives. In 7th IET International Conference on Power Electronics, Machines and Drives (PEMD 2014), 8-10 April 2014. 2014. Stevenage, UK: IET.

[18] Luri, S., I. Etxeberria-Otadui, A. Rujas, et al. Design of a supercapacitor based storage system for improved elevator applications. In 2010 IEEE Energy Conversion Congress and Exposition (ECCE), 12-16 Sept. 2010. 2010. Piscataway, NJ, USA: IEEE.

[19] Jinping, H., M. Chengxiong, L. Jiming, et al., Design and implementation of an energy feedback digital device used in elevator. IEEE Transactions on Industrial Electronics, 2011. 58(10): p. 4636-4642. 